\title{
Molecular imprinting ratiometric fluorescence sensor for highly selective and sensitive detection of phycocyanin
}

\author{
Xiaoyan Wang ${ }^{\mathrm{a}, \mathrm{b}, \mathrm{c}}$, Jialuo $\mathrm{Yu}^{\mathrm{a}, \mathrm{b}}$, Qi Kang ${ }^{\mathrm{a}}$, Dazhong Shen ${ }^{\mathrm{a}, *}$, Jinhua $\mathrm{Li}^{\mathrm{b}}$, Lingxin Chen ${ }^{\mathrm{b}, *}$ \\ ${ }^{a}$ College of Chemistry, Chemical Engineering and Materials Science, Collaborative Innovation Center of Functionalized Probes for Chemical Imaging in \\ Universities of Shandong, Key Laboratory of Molecular and Nano Probes, Ministry of Education, Shandong Provincial Key Laboratory of Clean Production of \\ Fine Chemicals, Shandong Normal University, Jinan 250014, China \\ ${ }^{\mathrm{b}}$ Key Laboratory of Coastal Environmental Processes and Ecological Remediation, Yantai Institute of Coastal Zone Research, Chinese Academy of Sciences, \\ Yantai 264003, China \\ c School of Pharmacy, Binzhou Medical University, Yantai 264003, China
}

\section{A R T I C L E I N F O}

\section{Article history:}

Received 10 August 2015

Received in revised form

30 September 2015

Accepted 7 October 2015

Available online 9 October 2015

Keywords:

Molecular imprinting

Ratiometric fluorescence

Fluorescence resonance energy transfer

Phycocyanin

\begin{abstract}
A B S T R A C T
A facile strategy was developed to prepare molecular imprinting ratiometric fluorescence sensor for highly selective and sensitive detection of phycocyanin (PC) based on fluorescence resonance energy transfer (FRET), via a sol-gel polymerization process using nitrobenzoxadiazole (NBD) as fluorescent signal source. The ratio of two fluorescence peak emission intensities of NBD and PC was utilized to determine the concentration of $\mathrm{PC}$, which could effectively reduce the background interference and fluctuation of diverse conditions. As a result, this sensor obtained high sensitivity with a low detection limit of $0.14 \mathrm{nM}$ within $6 \mathrm{~min}$, and excellent recognition specificity for PC over its analogues with a high imprinting factor of 9.1. Furthermore, the sensor attained high recoveries in the range of $93.8-110.2 \%$ at three spiking levels of PC, with precisions below $4.7 \%$ in seawater and lake water samples. The developed sensor strategy demonstrated simplicity, reliability, rapidity, high selectivity and high sensitivity, proving to be a feasible way to develop high efficient fluorescence sensors and thus potentially applicable for ultratrace analysis of complicated matrices.
\end{abstract}

(c) 2015 Elsevier B.V. All rights reserved.

\section{Introduction}

Eutrophication of inland lakes, reservoirs, estuaries, and coastal waters, is becoming a frequent phenomenon, which has received increasing attention due to its harmful impacts on the aquatic environment (Sun et al., 2015; Mishra and Mishra, 2014). Cyanobacterial blooms, as one of distinct types of eutrophication, have posed a serious threat to both the environment and humans, particularly under blooming conditions (Sun et al., 2015; Lyu et al., 2013). Phycocyanin (PC), an accessory cyanobacteria-specific pigment emitting red light with a maximum at about $650 \mathrm{~nm}$, can provide effective indication to assess cyanobacterial blooms (Qi et al., 2014). Hence, identifying and quantifying PC by developing cost effective, rapid, facile and applicable methods is highly crucial.

Currently, fluorescent detection has become a powerful tool due to its high sensitivity, simplicity and low background signal. Thus, fluorescence materials have recently aroused wide concerns for detecting trace amounts of analytes (Yu et al., 2014). Up to now, most fluorescent materials employ a sole responsive signal for

\footnotetext{
* Corresponding authors.

E-mail addresses: dzshen@sdnu.edu.cn (D. Shen), lxchen@yic.ac.cn (L. Chen).
}

detection, which can be readily perturbed by the change in fluorescence intensity and some external factors including environmental and instrumental efficiency (Koneswaran and Narayanaswamy, 2012). Fortunately, ratiometric measurement has the advantages to eliminate environmental effects and to give more precise measurement by its self-referencing of two emission bands, since it uses the ratio of two fluorescent peaks instead of the absolute intensity of one peak (Liu et al., 2013). Owing to the improved sensitivity, ratiometric fluorescence technique has attracted increasing attention for trace level analysis. For example, Wang's group proposed several ratiometric probes for the visual observation of fluorescence color changes induced by analytes without the need of elaborate equipment (Yao et al., 2013; Zhang et al., 2014; Zhu et al., 2014). Zhang's group developed a nanohybrid ratiometric fluorescence sensor for selective detection of mercuric ions. The sensor can effectively identify $\mathrm{Hg}^{2+}$ with the detection limit as low as 42 nM (Lan et al., 2014). However, as for most probes/sensors, selectivity is still very challenging especially for detection of trace analytes in complicated matrices.

Meanwhile, molecular imprinting technology (MIT) known as a technique for creation of tailor-made binding sites in a polymer matrix, i.e., molecularly imprinted polymers (MIPs), has attracted great concerns (Chen et al., 2011; Fu et al., 2015). The introduction 
of MIT into the design of fluorescence probes/sensors strategies will combine the advantages of high selectivity of MIPs with high sensitivity of fluorescence. So, it is naturally conceivable to develop molecular imprinting based fluorescence strategies with high selectivity and sensitivity for detection of low content target analytes from complex samples. However, as far as we know, there are few examples on molecular imprinting based ratiometric fluorescence probes/sensors. Recently, a kind of MIP coated quantum dot (MIP@QD) fluorescence sensor was reported, which combined with ratiometric fluorescence techniques to detect 2,4,6-trinitrotoluene (Xu and Lu, 2015). The assay platform exhibited excellent selectivity and sensitivity with a detection limit of $15 \mathrm{nM}$.

Usually, there are mainly two methods used to synthesize fluorescence-based MIPs. One method is to synthesize functional monomers with fluorophore, such as pyrene derivative (Chen et al., 2014) and 1,8-naphthalimide dye (Rouhani and Nahavandifard, 2014), by monitoring the change of fluorescence spectrum from the combination of the analytes with fluorescent functional monomer. However, the method often needs complicated synthesis procedure for fluorescence functional monomer. The other method is embedding fluorescent signal into MIPs and detecting analytes by fluorescence spectroscopy (Liu et al., 2011). Among the optical materials investigated, QDs (Li et al., 2013; Tan et al., 2013; Zhou et al., 2014; Zhang et al., 2015) and organic fluorescent compounds (Descalzo et al., 2013) are the most widely used fluorescent signal sources. Similarly, the process is rather laborious and time-consuming. Therefore, to prepare fluorescence-based MIPs with a more labor-saving process and higher efficiency is of extreme significance and greatly desirable.

Herein, inspired by these studies, through a simple, time-saving and facile sol-gel polymerization process, we developed a novel molecular imprinting ratiometric fluorescence sensor for selective and sensitive detection of PC on the basis of fluorescence resonance energy transfer (FRET). Organic dye nitrobenzoxadiazole (NBD) was chosen as fluorescent donor, because of its three features: its emission spectrum partially overlapping with the absorption spectrum of PC for FRET, its high fluorescence efficiency for fluorescent detection, and its easy reaction with 3-aminopropyltriethoxysilane (APTES) for being easily attached onto the surface of silica particles. The obtained fluorescence sensor was well characterized and its analytical performances were investigated in detail. The potential application of the sensor to real samples including seawater and lake water was also explored.

\section{Experimental}

\subsection{Reagents and materials}

3-Aminopropyltriethoxysilane (APTES), bovine serum albumin (BSA), and 4-chloro-7-nitro-2,1,3-benzoxadiazole (NBD-Cl) were purchased from Sigma-Aldrich (Shanghai, China). Ammonium hydroxide (25\%), absolute ethanol, methanol, acetic acid (HAc), tetraethoxysilicane (TEOS) and phosphate buffered saline (PBS) were supplied by Sinopharm Chemical Reagent Co. Ltd. (Shanghai, China). Phycocyanin (PC), phycoerythrin (PE), and spirulina powder were kindly provided by Shandong Oriental Ocean Co. (Yantai, China). The PC obtained from spirulina has the molecular weight of $110 \mathrm{kDa}$ containing two subunits, and its isoelectric point (pI) is 4.3 , and its main functional groups are carboxyl and amino groups.

\subsection{Characterization}

Fluorescence measurements were performed on a Fluoromax-4 Spectrofluorometer (Horiba Scientific). UV-vis spectra were measured on a Thermo Scientific NanoDrop 2000/2000C spectrophotometer (Thermo Fisher Scientific, Waltham, MA). The morphological evaluation was examined on a scanning electron microscope (SEM, Hitachi S-4800 FE-SEM, operating at $5 \mathrm{kV}$ ) and transmission electron microscopy (TEM, JEM-1230, operating at $100 \mathrm{kV}$ ). FT-IR analyses were carried out by a FT-IR spectrometer (Thermo Nicolet Corporation, USA).

\subsection{Synthesis of APTES-NBD conjugates}

The APTES-NBD conjugates were synthesized according to the reported method (Geng et al., 2010). $11.8 \mathrm{mg}$ NBD-Cl was first dissolved in $10 \mathrm{~mL}$ anhydrous ethanol containing APTES $(0.5 \mathrm{~mL}$; molar ratio of NBD/APTS 1:40) with stirring in a dry nitrogen atmosphere. After $12 \mathrm{~h}$ of reaction in the dark, the APTES-NBD conjugates were formed in the mixture solution, which would be used as donor precursor.

\subsection{Preparation of $\mathrm{SiO}_{2} @ N B D @ M I P s$}

The $\mathrm{SiO}_{2} @$ NBD@MIPs were prepared through a sol-gel process. $\mathrm{SiO}_{2}$ nanoparticles were firstly synthesized according to the report (Gao et al., 2010). Subsequently, $160 \mu \mathrm{L}$ of the above APTES-NBD conjugates solution and $20 \mathrm{mg}$ of PC were added to $30 \mathrm{~mL}$ of ultrapure water containing $120 \mathrm{mg} \mathrm{\textrm {SiO } _ { 2 }}$ nanoparticles. After stirred for $30 \mathrm{~min}, 200 \mathrm{~mL}$ of $\mathrm{NH}_{3} \cdot \mathrm{H}_{2} \mathrm{O}$ and $200 \mu \mathrm{L}$ of TEOS were added and kept stirring overnight in the dark. Finally, the products were centrifuged and washed with methanol/HAc $(9: 1, \mathrm{v} / \mathrm{v})$ for three times to remove PC, and then the resultant $\mathrm{SiO}_{2} @ \mathrm{NBD} @ M I P s$ (MIPs, for simplicity) were dispersed in $10 \mathrm{~mL}$ ultrapure water for further use. As a control, their corresponding non-imprinted polymers $\mathrm{SiO}_{2} @ N B D @ N I P s$, namely NIPs for simplicity, were synthesized under the identical conditions in the absence of the template PC.

\subsection{Fluorescence measurement}

All the fluorescence (FL) intensity detections were conducted under the same conditions: the excitation and emission slit widths were both $7 \mathrm{~nm}$ and the excitation wavelength was set at $468 \mathrm{~nm}$ with a recording emission range of 488-700 nm. SiO ${ }_{2} @ N B D @ M I P s$ or $\mathrm{SiO}_{2} @ N B D @ N I P s$ was added to the known concentration of analyte and the final concentration of MIPs or NIPs was $0.9 \mathrm{mg} \mathrm{mL}^{-1}$.

\subsection{Analysis of water samples}

Samples including seawater and lake water were used to demonstrate the applicability of the $\mathrm{SiO}_{2} @ N B D @ M I P s$ for the detection of PC. Seawater samples were randomly collected from the surface seawater of Yellow Sea and lake water samples were collected from San Yuan Lake, located in the coastal zone of Yantai City. Before analysis, the water samples were filtered with $0.45 \mu \mathrm{m}$ microfiltration membrane to remove the suspended particles. The spiked seawater and lake water samples diluted 100-fold with different concentrations of PC were used to validate the accuracy and practicality of the MIPs.

\section{Results and discussion}

\subsection{Preparation and detection principle of $\mathrm{SiO}_{2} @ N B D @ M I P s$}

Scheme 1 illustrates the preparation process and possible detection principle of $\mathrm{SiO}_{2} @ N B D @ M I P s$ sensor. As seen, the donor precursor (APTES-NBD) was synthesized via the reaction of NBD$\mathrm{Cl}$ with APTES by nucleophilic substitution. Then, $\mathrm{SiO}_{2}$ 


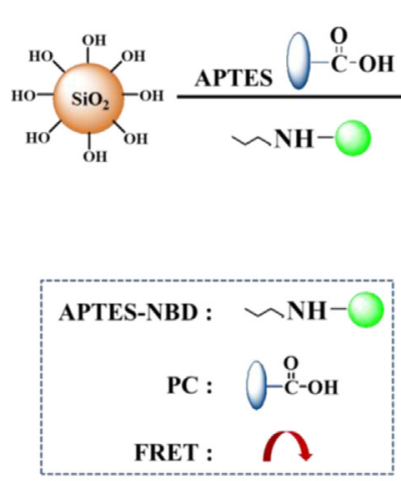
$\frac{\text { TEOS }}{\mathrm{NH}_{3} \cdot \mathrm{H}_{2} \mathrm{O}}$
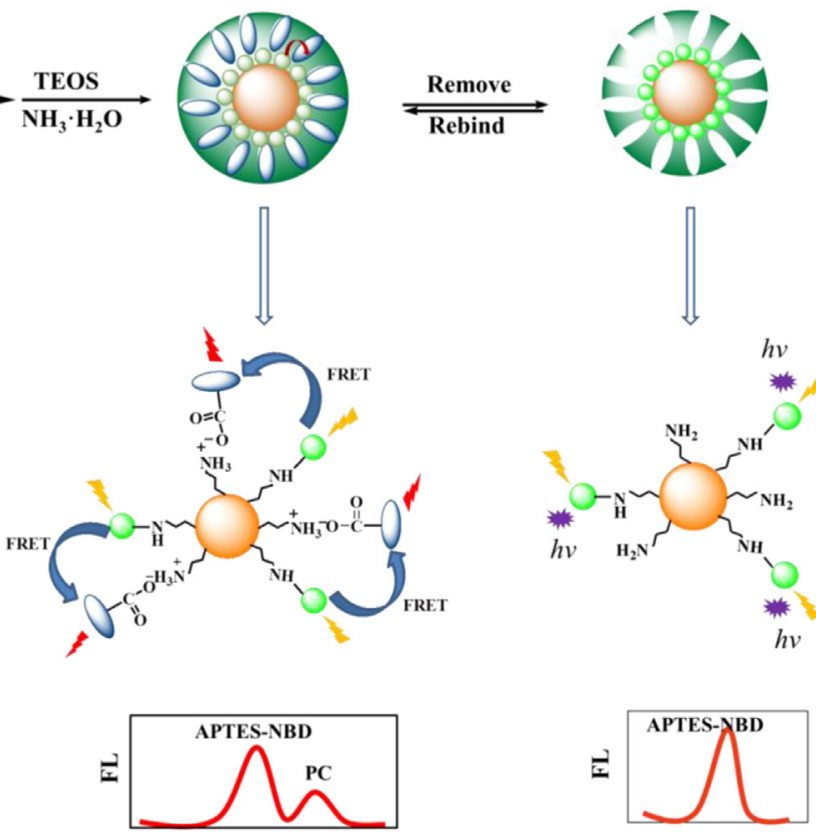

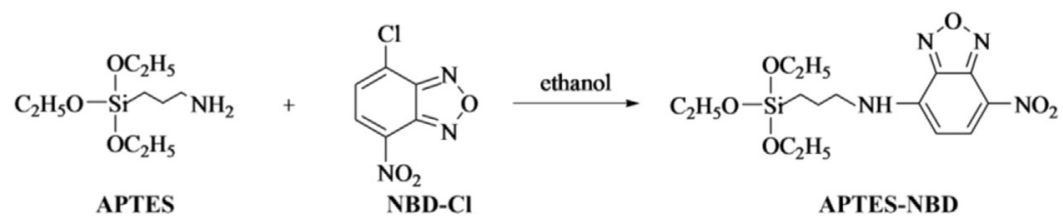

Scheme 1. Schematic illustration for the preparation process and possible detection principle of $\mathrm{SiO}_{2} @ \mathrm{NBD}_{\mathrm{N}} \mathrm{MIPs}$.

nanoparticles were used as core materials, PC as the template molecule and TEOS as the cross linker, and the resultant APTESNBD conjugates as donor precursor and APTES as functional monomers were spontaneously anchored onto the surface of the silica nanoparticles through a condensation reaction, which led to the formation of $\mathrm{SiO}_{2} @ \mathrm{NBD} @$ MIPs after removing the embedded template PC. For this $\mathrm{SiO}_{2} @ N B D @ M I P s$ sensor, upon light irradiation, there is only the emission spectrum of NBD in the absence of $\mathrm{PC}$, however, in the presence of PC, the binding through hydrogen bond between the carboxyl group of $\mathrm{PC}$ and the amino group of APTES, will quench the NBD fluorescence and increase PC fluorescence emission through FRET, realizing ratiometric detection for PC.

Generally, FRET process requires the overlap of the donor emission spectrum of a fluorescent dye and the acceptor absorption spectrum of an analyte (or its derivatives) (Liu et al., 2013). In this study, APTES-NBD conjugates served as energy donor and PC as acceptor. As seen from Fig. S1A, the fluorescence emission spectrum of APTES-NBD conjugates overlapped the absorption spectrum of $\mathrm{PC}$, which suggested that the resonance energy transfer from the APTES-NBD conjugates to PC may occur if they were spatially close to each other. The fluorescence emission of APTES-NBD conjugates can be strongly absorbed by PC, resulting in fluorescence quenching of the donor. Actually, the PC binding will quench the fluorescence of donor by two possible pathways: the resonance energy transfer and the common charge transfer between donor and PC. Fig. S1B demonstrates the quenching mechanism of donor fluorescence by the resonance energy transfer from donor to PC acceptor. When the excited-state electrons of the donor return to ground state through the pathway of $\mathrm{a}, \mathrm{b}$, and $\mathrm{c}$, the ground-state electrons of the PC will transit to the excited states through the corresponding pathway of $a^{\prime}, b^{\prime}$, and $c^{\prime}$, respectively, due to the polar-polar resonance of donor and acceptor. The coupled transitions will lead to the highly efficient quenching of donor fluorescence (Gao et al., 2008). On the other hand, it has to be noted that the fluorescence of donor may also be quenched by the charge transfer, as illustrated in Fig. S1C. PC has many amino groups and the amino group has a nitrogen lone-pair electron, while NBD has an electron-deficient aromatic ring with a nitro. When the absorption band of PC (at $620 \mathrm{~nm}$ ) overlaps with the emission band of APTES-NBD conjugates (at $535 \mathrm{~nm}$ ), a charge transfer interaction may occur between electron-rich amino group of PC and the electron-deficient aromatic ring of NBD. However, in the case of PC, the resonance energy transfer is a predominant quenching process. Hence, in the presence of $\mathrm{PC}$, the excited-state energy of the donor APTES-NBD conjugates could be transferred to PC (Fig. S1B), leading to the reduction of donor fluorescence and the increase of acceptor fluorescence. Therefore, the as-prepared $\mathrm{SiO}_{2} @ N B D @ M I P s$ sensor could realize ratiometric fluorescence detection of PC based on FRET.

\subsection{Characterization of $\mathrm{SiO}_{2} @ N B D @ M I P s$}

The morphological structures of $\mathrm{SiO}_{2}, \mathrm{SiO}_{2} @ N B D @ M I P s$ and $\mathrm{SiO}_{2} @$ NBD@NIPs were characterized by TEM and SEM, as shown in Fig. 1. It was observed from Fig. $1 \mathrm{~A}$ that $\mathrm{SiO}_{2}$ nanoparticles had good dispersion with the average diameter about 70-90 nm. As seen from Fig. 1B, MIPs also exhibited good dispersion and the imprinting shell thickness was about 5-10 nm calculated from the TEM image. Noticeably, the MIPs displayed a highly rough outside polymeric surface (Fig. 1C), and in contrast, the surface of NIP particles was still smooth (Fig. 1D), which indicated that there are a large number of exposed complementary imprinted cavities on the surface of MIPs while no recognition sites on the surface of NIPs.

The UV absorption and fluorescence spectra were employed for 

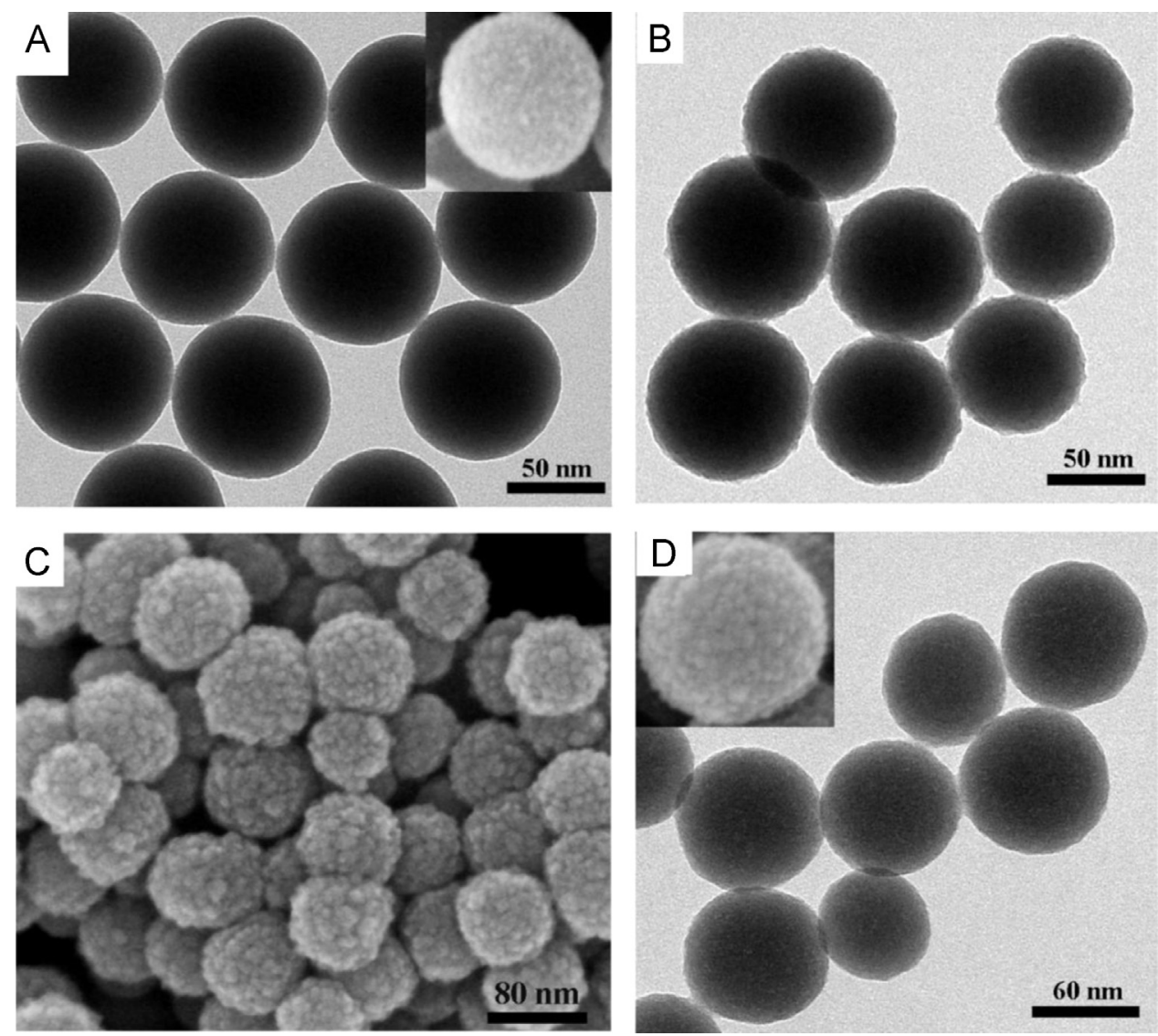

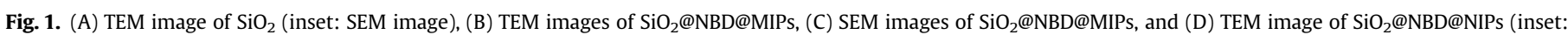
SEM image).

characterization of APTES-NBD conjugates. As known, the NBD-Cl, as an amine reactive reagent, is not fluorescent until it is covalently linked (Carlson et al., 2006). As seen from Fig. S2, the NBD-Cl has no absorption spectrum at $468 \mathrm{~nm}$ (a), however, after it reacted with APTES, new absorption spectrum at $468 \mathrm{~nm}$ emerged (b) as well as the fluorescence spectrum at $535 \mathrm{~nm}$ (d), indicating the APTES-NBD conjugates were successfully obtained. Moreover, the absorption spectrum of the $\mathrm{SiO}_{2} @ N B D @ M I P s$ at $468 \mathrm{~nm}$ (c) and its fluorescence spectra at $535 \mathrm{~nm}$ (e) further confirmed the successful modification of the APTES-NBD conjugates onto the surface of the silica nanoparticles through sol-gel process.

The infrared spectra of $\mathrm{SiO}_{2}$ (a) and MIPs (b) were displayed in Fig. S3. As can be seen, the characteristic peaks around 466 and $802 \mathrm{~cm}^{-1}$ could be ascribed to Si-O stretching vibration, and the wide and strong absorption band around $1095 \mathrm{~cm}^{-1}$ belonged to stretching vibrations of $\mathrm{Si}-\mathrm{O}-\mathrm{Si}$, illustrating the presence of $\mathrm{SiO}_{2}$ matrices in the two materials. The characteristic peak of amino groups at around $1388 \mathrm{~cm}^{-1}$, verified the successful introduction of amine ligands at the surface of silica nanoparticles, and we can judge the successful preparation of $\mathrm{SiO}_{2} @ A P T E S-N B D @ M I P s$ instead of $\mathrm{SiO}_{2} @ A P T E S$, since the APTES-NBD conjugates had been evidenced as above UV and fluorescence spectra.

\subsection{Fluorescence properties of the $\mathrm{SiO}_{2} @ N B D @ M I P s$}

Fluorescence performances of the $\mathrm{SiO}_{2} @ \mathrm{NBD} @ \mathrm{MIPs}$ sensor for PC strongly depended on the variables including $\mathrm{pH}$, response time and the fluorescence stability. And the relevant variables were studied systematically as follows. The effect of solution acidity on the $\mathrm{SiO}_{2} @$ NBD@MIPs response to PC has been discussed, since $\mathrm{pH}$ played an important influence on the charge of PC and the fluorescence intensity of MIPs. Experimental results showed that the $I_{657} / I_{535}$ ratio of MIPs performed well in the $\mathrm{pH}$ range from 4 to 8.5 (Fig. 2A). Under strong acidic conditions $(\mathrm{pH}<4)$, the amino groups on the surface of MIPs may be partially protonated, which decreased the electrostatic interaction of MIPs and PC. When $\mathrm{pH}$ was higher than 8.5, PC may be slightly denatured, which may decrease the interaction between the PC and the imprinted cavities. It can be clearly seen that the $I_{657} / I_{535}$ ratio of MIPs increased with the increase of $\mathrm{pH}$ from 4 to 6 and then decreased when $\mathrm{pH}$ values were higher. This revealed that $\mathrm{pH} 6$ was the most suitable condition for detection of PC, which was selected for further experiments.

The response time of the MIPs was also investigated to assess the accessibility to binding sites. As seen from Fig. 2B, the fluorescence intensity at $535 \mathrm{~nm}$ decreased fast within 6 min when the concentration of PC was $100 \mathrm{nM}$, after which the curve became flat. Therefore, 6 min was chosen as the response time for further experiments. The response time of the MIPs is short, which can be attributed to the thin imprinting layer. As can be seen from the TEM image of $\mathrm{SiO}_{2} @$ NBD@MIPs, after sol-gel polymerization process, the imprinting layer on the surface of $\mathrm{SiO}_{2}$ is only several nanometres, which provides low mass transfer resistance, high site accessibility toward the template PC, resulting in the rapid response of the MIPs for PC. As is well known that, reducing the imprinting-shell layer thickness is an effective way to shorten the response time of MIPs for template. Moreover, the fluorescence stability of $\mathrm{SiO}_{2} @ \mathrm{NBD} @$ MIPs was also investigated during the storage by repeatedly detecting the fluorescence intensity every day. The intensity retained $98 \%$ of its initial response when the MIPs were stored for 10 days (Fig. 2B, inset), which implied that the resultant MIPs displayed good physical stability and chemical 
A

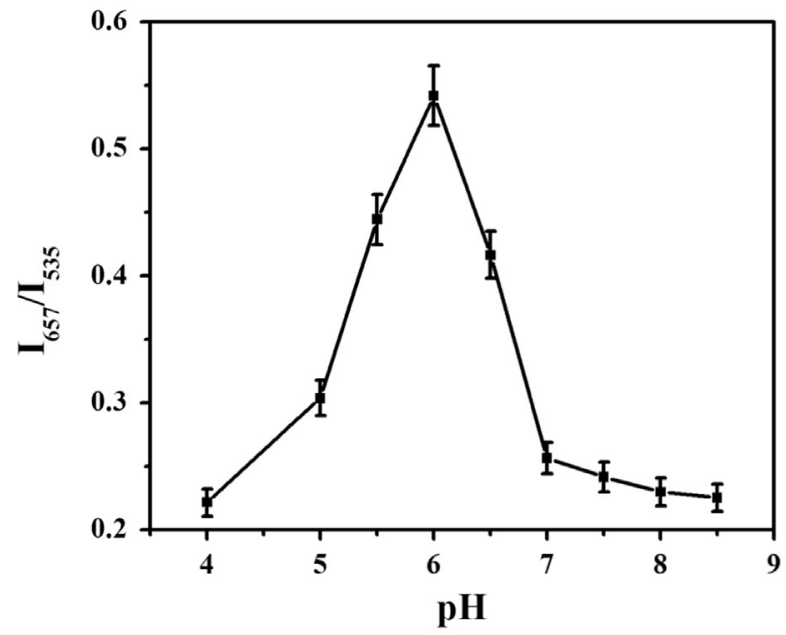

B

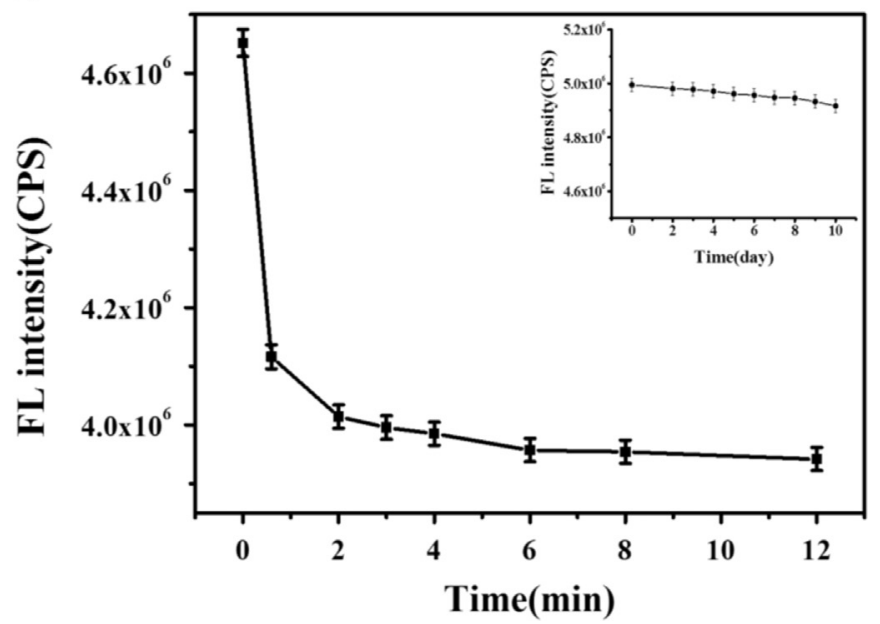

Fig. 2. (A) Effect of $\mathrm{pH}$ on the $I_{657} / I_{535}$ ratio of $\mathrm{SiO}_{2} @ N B D @ M I P s$ in the presence of $100 \mathrm{nM}$ PC at room temperature, (B) fluorescence response time of $\mathrm{SiO}_{2}$ @NBD@MIPs for PC (inset was fluorescence intensity change of $\mathrm{SiO}_{2} @ \mathrm{NBD} @ M I P s$ within 10 days).

inertness.

\subsection{Sensitivity and selectivity of the sensor}

To evaluate the sensitivity of the $\mathrm{SiO}_{2} @ N B D @ M I P s$ sensor, fluorescence responses were measured upon addition of different amounts of PC in water dispersion and a working curve was established by plotting the ratio of the emission intensities at $535 \mathrm{~nm}$ and $657 \mathrm{~nm}\left(I_{657} / I_{535}\right)$ versus PC concentration (Fig. 3A). It can be seen that in the absence of PC, excitation of the MIPs at $468 \mathrm{~nm}$ could only result in the emission of the donor APTES-NBD conjugates at $535 \mathrm{~nm}$, and no characteristic emission of PC acceptor was observed. With the addition of PC, the APTES-NBD emission at $535 \mathrm{~nm}$ gradually decreased, and the emission band at $657 \mathrm{~nm}$ appeared and increased remarkably, which corresponded to the fluorescence emission of PC, indicating that the energy of the donor APTES-NBD conjugates could be transferred to PC. By plotting the emission intensity ratio versus PC concentration, the calibration curve for the MIPs was obtained (inset of Fig. 3A). As seen from the figure, the ratio of the intensities at $657 \mathrm{~nm}$ to that at $535 \mathrm{~nm}\left(I_{657} / I_{535}\right)$ increased steadily with PC concentration increasing and the linear range was 1-250 nM with the detection limit (LOD, $\mathrm{S} / \mathrm{N}=3$ ) of $0.14 \mathrm{nM}$, owing to the two well-resolved
A

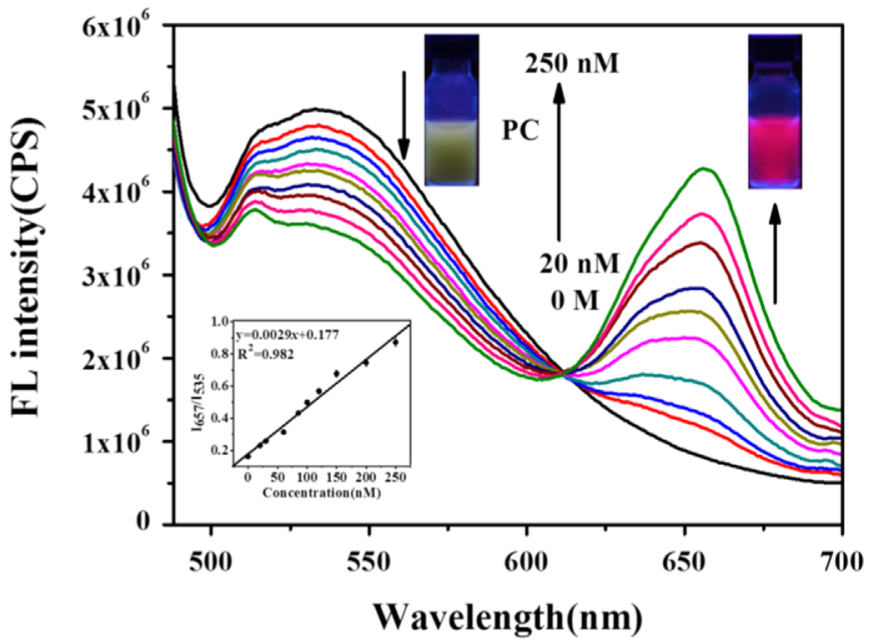

B

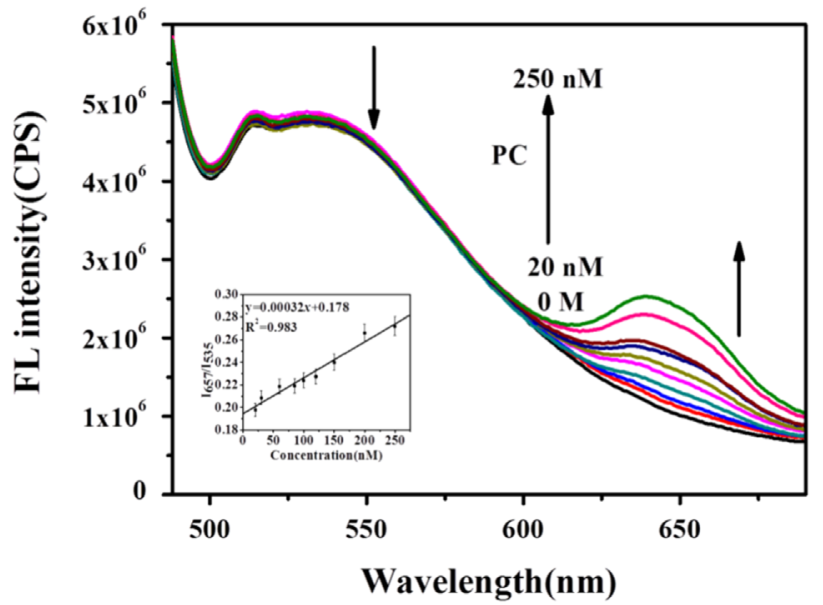

Fig. 3. The fluorescence spectra of the ratiometric sensor with addition of the different concentrations of PC for the MIPs (A) and for the NIPs (B). Concentrations of PC from left to right are $0,20,30,60,85,100,120,150,200$ and $250 \mathrm{nM}$. Concentration of MIPs or NIPs was $0.9 \mathrm{mg} \mathrm{mL}^{-1}$; excited light, $468 \mathrm{~nm}$; silt widths of excitation and emission, $7 \mathrm{~nm}$. Inset shows the linear relations between $I_{657} / I_{535}$ and the concentrations of PC from 0 to $250 \mathrm{nM}$.

emission peaks with comparable fluorescence intensities. Meanwhile, the fluorescence property of the non-imprinted $\mathrm{SiO}_{2}$ @NBD@NIPs to PC was also examined. From Fig. 3B, we can see that fluorescence intensity of NIPs was slightly decreased with the addition of PC, and hence, the sensitivity was much lower and the linear range was narrower (inset of Fig. 3B). As observed from Fig. 3, MIPs and NIPs offered different linear relationships, and the decrease of fluorescence intensity of MIPs was much larger than that of the NIPs at the same PC concentration. So, a high imprinting factor could be obtained of 9.1. For the MIPs, most of the recognition sites were located on the surface of silica matrix, so PC has more chance to enter the recognition sites, quenching the fluorescence intensity of the donor APTES-NBD conjugate obviously and increasing the fluorescence intensity of PC. As for NIPs, there are no recognition sites on the surface of silica matrix, so PC cannot enter into the inner of the NIPs, resulting in slight variation of the fluorescence intensity. And the fluorescence intensity of the donor can only be quenched by non-specific adsorption of PC. The results further demonstrated that the MIPs based fluorescence sensor could sensitively detect PC.

The selectivity of MIPs sensor was further evaluated by a competitive binding test using PE, spirulina powder and BSA. PE is 


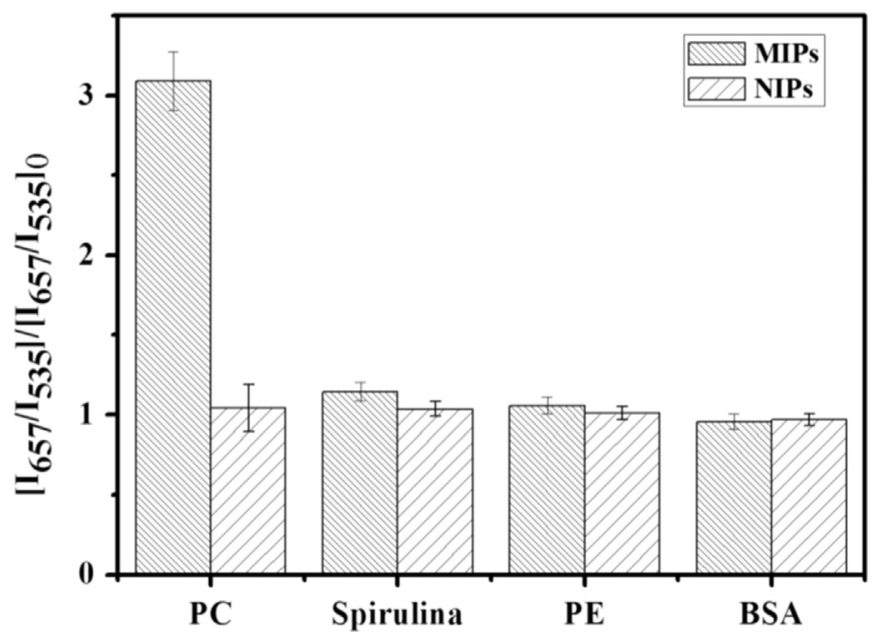

Fig. 4. The selectivity of MIPs and NIPs for PC over other analogues including spirulina, PE and BSA at concentration of $11 \mathrm{mg} \mathrm{L}^{-1}$. Concentration of MIPs or NIPs was $0.9 \mathrm{mg} \mathrm{mL}^{-1}$; excited light, $468 \mathrm{~nm}$; silt widths of excitation and emission, $7 \mathrm{~nm}$.

Table 1

Results for the determination of the PC in seawater and lake water samples.

\begin{tabular}{llll}
\hline Sample & Added $(\mathrm{nM})$ & Found $(\mathrm{nM})$ & Recovery $^{\mathrm{a}} \pm$ RSD $(\%)$ \\
\hline Seawater & 0 & 0 & - \\
& 20.00 & 19.78 & $98.9 \pm 3.4$ \\
& 50.00 & 53.70 & $107.4 \pm 3.8$ \\
& 100.00 & 109.54 & $109.5 \pm 4.1$ \\
Lake water & 0 & & \\
& 20.00 & 0 & - \\
& 50.00 & 18.76 & $93.8 \pm 3.5$ \\
& 100.00 & 52.03 & $104.1 \pm 4.2$ \\
& & 110.20 & $110.2 \pm 4.7$ \\
\hline
\end{tabular}

${ }^{a}$ Average value from three individual experiments.

the analogue to PC and spirulina powder contains amounts of PC and PE. In order to contrast, we also used BSA with different shapes and molecular weights from PC as a competitive protein. It can be seen from Fig. 4 that PC induced a prominent fluorescence ratio $\left(I_{657} / I_{535}\right)$ increment compared to the blank sample (no protein was added). In contrast, addition of other proteins led to smaller or no fluorescence ratio change, because the recognition sites of PC imprinted cavities were not complementary to them, resulting in less chance to enter the recognition sites. And it can also be seen that the fluorescence ratio of the spirulina is higher than that of PE, since spirulina powder contains certain amounts of PC. The results implied that the MIPs had a high selectivity for PC over other competitive proteins.

\subsection{Practical application of the sensor to environmental water samples}

To further evaluate its applicability in real water samples, the MIPs ratiometric fluorescence sensor was applied to detect PC in real water samples diluted 100 -fold spiked with different amounts of PC, including seawater and lake water. Studies were carried out with three concentrations $(25,50$ and $100 \mathrm{nM})$ spiked in each sample. Each concentration was done in triplicate, and the averages were presented with relative standard deviation (RSD). As listed in Table 1, satisfactory recoveries were attained of 98.9$109.5 \%$ with RSDs of $3.4-4.1 \%$ for the spiked seawater samples and
93.8-110.2\% with RSDs of $3.5-4.7 \%$ for the spiked lake water samples, respectively. The results indicated that the MIPs ratiometric fluorescence sensor was practically feasible for accurate determination of trace PC in complicated environmental water samples, and consequently algae blooms monitoring and removal/ remediation could be successfully performed.

\section{Conclusions}

In summary, we have successfully constructed a novel PC imprinted ratiometric fluorescence sensor via facile sol-gel polymerization for the recognition and detection of PC based on FRET. By taking advantages of the high selectivity of MIPs and the high sensitivity of ratiometric fluorescence, the sensor demonstrated highly selective and sensitive recognition and determination of PC. The simple, rapid, reliable, time-saving and cost-effective strategy provided an excellent fluorescent analysis platform for PC monitoring. Moreover, this strategy may provide an alternative approach for constructing FRET-based detection systems for sensitively and selectively recognizing and detecting a variety of analytes for potential utilizations.

\section{Acknowledgments}

This work was financially supported by the National Natural Science Foundation of China (21175084, 21275091, 21275158, and 21477160), the Research Fund for the Doctoral Program of Higher Education of China (20113704110003), and the Scientific Research Foundation for the Returned Overseas Chinese Scholars, State Education Ministry.

\section{Appendix A. Supplementary material}

Supplementary data associated with this article can be found in the online version at http://dx.doi.org/10.1016/j.bios.2015.10.019.

\section{References}

Carlson, C.A., Lloyd, J.A., Dean, S.L., Walker, N.R., Edmiston, P.L., 2006. Anal. Chem. 78, 3537-3542.

Chen, L.X., Xu, S.F., Li, J.H., 2011. Chem. Soc. Rev. 40, $2922-2942$.

Chen, Z.H., Alvarez-Perez, M., Navarro-Villoslada, F., Moreno-Bondi, M.C., Orellana, G., 2014. Sens. Actuators B 191, 137-142.

Descalzo, A.B., Somoza, C., Moreno-Bondi, M.C., Orellana, G., 2013. Anal. Chem. 85, 5316-5320.

Fu, J.Q., Chen, L.X., Li, J.H., Zhang, Z., 2015. J. Mater. Chem. A 3, 13598-13627.

Gao, D.M., Wang, Z.Y., Liu, B.H., Ni, L., Wu, M.H., Zhang, Z.P., 2008. Anal. Chem. 80, $8545-8553$.

Gao, R.X., Zhang, J.J., He, X.W., Chen, L.X., Zhang, Y.K., 2010. Anal. Bioanal. Chem. 398, 451-461.

Geng, J.L., Liu, P., Liu, B.H., Guan, G.J., Zhang, Z.P., Han, M.Y., 2010. Chem. Eur. J. 16, 3720-3727.

Koneswaran, M., Narayanaswamy, R., 2012. Microchim. Acta 178, 171-178.

Lan, M.H., Zhang, J.F., Chui, Y.S., Wang, P.F., Chen, X.F., Lee, C.S., Kwong, H. Lun, Zhang, W.J., 2014. ACS Appl. Mater. Interfaces 6, 21270-21278.

Liu, B.Y., Zeng, F., Wu, S.Z., Wang, J.S., Tang, F.C., 2013. Microchim. Acta 180, $845-853$.

Li, D.Y., He, X.W., Chen, Y., Li, W.Y., Zhang, Y.K., 2013. ACS Appl. Mater. Interfaces 5, 12609-12616.

Liu, R., Guan, G., Wang, S., Zhang, Z., 2011. Analyst 136, 184-190.

Lyu, H., Wang, Q., Wu, C.Q., Zhu, L., Yin, B., Li, Y.M., Huang, J.Z., 2013. Ecol. Inform. 18, $178-187$.

Mishra, S., Mishra, D.R., 2014. Environ. Res. Lett. 9, 114003-114012.

Qi, L., Hu, C.M., Duan, H.T., Cannizzaro, J., Ma, R.H., 2014. Remote Sens. Environ. 154, 298-317.

Rouhani, S., Nahavandifard, F., 2014. Sens. Actuators B 197, 185-192.

Sun, D.Y., Hu, C.M., Qiu, Z.F., Shi, K., 2015. Opt. Express 23, 3055-3074.

Tan, L., Kang, C., Xu, S., Tang, Y., 2013. Biosens. Bioelectron. 48, 216-223.

Xu, S.F., Lu, H.Z., 2015. Chem. Commun. 51, 3200-3203. 
Yao, J.L., Zhang, K., Zhu, H.J., Ma, F., Sun, M.T., Yu, H., Sun, J., Wang, S.H., 2013. Anal. Chem. 85, 6461-6468.

Yu, F.B., Han, X.Y., Chen, L.X., 2014. Chem. Commun. 50, 12234-12249.

Zhang, K., Yu, T., Liu, F., Sun, M.T., Yu, H., Liu, B.H., Zhang, Z.P., Jiang, H., Wang, S.H., 2014. Anal. Chem. 86, 11727-11733.
Zhang, Z., Li, J.H., Wang, X.Y., Shen, D.Z., Chen, L.X., 2015. ACS Appl. Mater. Interfaces 7, 9118-9127.

Zhou, Y., Qu, Z., Zeng, Y., Zhou, T., Shi, G., 2014. Biosens. Bioelectron. 52, 317-323.

Zhu, H.J., Yu, T., Xu, H.D., Zhang, K., Jiang, H., Zhang, Z.P., Wang, Z.Y., Wang, S.H., 2014. ACS Appl. Mater. Interfaces 6, 21461-21467. 